\title{
Transition technologies towards 6G networks
}

\author{
Thiago R. Raddo ${ }^{1,2}$, Simon Rommel ${ }^{1}$, Bruno Cimoli ${ }^{*}$ (@), Chris Vagionas ${ }^{3}$, Diego Perez-Galacho ${ }^{4}$, \\ Evangelos Pikasis ${ }^{5}$, Evangelos Grivas ${ }^{6}$, Konstantinos Ntontin $^{6}$, Michael Katsikis $^{6}$, Dimitrios Kritharidis ${ }^{6}$, \\ Eugenio Ruggeri ${ }^{3}$, Izabela Spaleniak ${ }^{7}$, Mykhaylo Dubov ${ }^{7}$, Dimitrios Klonidis ${ }^{8}$, George Kalfas ${ }^{3}$, Salvador Sales ${ }^{4}$, \\ Nikos Pleros ${ }^{3}$ and Idelfonso Tafur Monroy ${ }^{1}$
}

*Correspondence:

b.cimoli@tue.nl

${ }^{1}$ Institute for Photonic

Integration, Eindhoven

University of Technology,

$5600 \mathrm{MB}$ Eindhoven, The

Netherlands

Full list of author information

is available at the end of the

article

\begin{abstract}
The sixth generation (6G) mobile systems will create new markets, services, and industries making possible a plethora of new opportunities and solutions. Commercially successful rollouts will involve scaling enabling technologies, such as cloud radio access networks, virtualization, and artificial intelligence. This paper addresses the principal technologies in the transition towards next generation mobile networks. The convergence of $6 \mathrm{G}$ key-performance indicators along with evaluation methodologies and use cases are also addressed. Free-space optics, Terahertz systems, photonic integrated circuits, softwarization, massive multiple-input multiple-output signaling, and multicore fibers, are among the technologies identified and discussed. Finally, some of these technologies are showcased in an experimental demonstration of a mobile fronthaul system based on millimeter 5G NR OFDM signaling compliant with 3GPP Rel. 15. The signals are generated by a bespoke $5 \mathrm{G}$ baseband unit and transmitted through both a $10 \mathrm{~km}$ prototype multi-core fiber and $4 \mathrm{~m}$ wireless $\mathrm{V}$-band link using a pair of directional $60 \mathrm{GHz}$ antennas with $10^{\circ}$ beamwidth. Results shown that the $5 \mathrm{G}$ and beyond fronthaul system can successfully transmit signals with both wide bandwidth (up to $800 \mathrm{MHz}$ ) and fully centralized signal processing. As a result, this system can support large capacity and accommodate several simultaneous users as a key candidate for next generation mobile networks. Thus, these technologies will be needed for fully integrated, heterogeneous solutions to benefit from hardware commoditization and softwarization. They will ensure the ultimate user experience, while also anticipating the quality-of-service demands that future applications and services will put on $6 \mathrm{G}$ networks.
\end{abstract}

Keywords: 5G, 6G, Key-performance indicator (KPI), mmWave, Free-space optics (FSO), Terahertz (THz), Softwarization, Virtualization, Backhaul, Fronthaul

\section{Introduction}

Mobile operators will soon be able to unlock many new business opportunities with $5^{\text {th }}$ generation (5G) and what comes after it, namely 6th generation (6G). Although 5G systems have not yet been rolled-out in several countries, there are already several works addressing 6G mobile systems [1-9], mainly focusing on emerging and transitional

(c) The Author(s), 2021. Open Access This article is licensed under a Creative Commons Attribution 4.0 International License, which permits use, sharing, adaptation, distribution and reproduction in any medium or format, as long as you give appropriate credit to the original author(s) and the source, provide a link to the Creative Commons licence, and indicate if changes were made. The images or other third party material in this article are included in the article's Creative Commons licence, unless indicated otherwise in a credit line to the material. If material is not included in the article's Creative Commons licence and your intended use is not permitted by statutory regulation or exceeds the permitted use, you will need to obtain permission directly from the copyright holder. To view a copy of this licence, visit http:// creativecommons.org/licenses/by/4.0/. 
technologies. But commercial success of these next generation systems is closely linked to a tight control of costs and flexibility in the evolution of network topology [10]. 6G networks are projected to allow most appliances, devices, and lamp posts to create their own wireless cell in order to connect other devices. This smaller cell will overlap seamlessly with classical cloud services. Softwarization is the abstraction of underlying hardware and physical components for innovative solutions based mainly on software and related functionalities. Softwarization and more dynamic allocation of resources on demand, are needed for new services or to expand existing capacity. Finally, artificial intelligence (AI) will also play a key role in next generation systems as part of solutions based on softwarization.

Next generation mobile networks will be heterogeneous, flexible, and support requirements for on-the-fly applications. This unique adaptive instantaneous service-delivery-on-demand needs to achieve ultra-low levels of latency as well as higher capacity. Today's consumer communications applications including high quality video streaming, web browsing, and voice over IP will become standard features in all future networks. Next generation mobile networks must support another order of magnitude of service provision. It should cope with unforeseen behavior of highly demanding applications that will eventually become key to the successful implementation and roll-out of $5 \mathrm{G}$ systems. Applications and services including autonomous last-mile delivery of general goods [11], fully interactive media entertainment and domestic robotic services need to be considered now.

A highly competitive race to roll-out the $5 \mathrm{G}$ mobile infrastructure is already underway. Many large businesses, stakeholders, and companies are rushing to establish their own proprietary solutions to establish a unique position in the highly competitive market. The expected roll-out timetable for $5 \mathrm{G}$ systems is already being implemented. The infrastructure is expected to be operational in the course of 2020. In Europe, the telecom market expects several 5G roll-outs in time for the EURO 2020 football championships, along with potential 5G support events in both stadiums and fan zones [12]. In turn, in order to address interoperability and seamless connection among different solutions and technologies, centralized or cloud radio access network (C-RAN) fronthaul architectures should be considered [13, 14]. Such a concept of fronthaul architecture, C-RAN, might overcome the differences among solutions, and further support dynamical network resource utilization and slicing. These are needed to increase the chances of successfully meeting 5G key-performance indicators (KPIs). The 5G C-RAN fronthaul can operate in the millimeter wave (mmWave) range [15] with multiple bands within the frequency range from 20 to $300 \mathrm{GHz}$ as part of a hybrid optical wireless solution. Some technologies operate within the V-band region $(\sim 60 \mathrm{GHz})$ whereas others operate within $\mathrm{K} / \mathrm{Ka}$ band ( 24.25 to $27.50 \mathrm{GHz})$, i.e., the 5G NR n258 band. Figure 1 illustrates examples of networking scenarios based on different signal coverage areas within mmWave bands. It is worth pointing out that each spectrum frequency band has its own benefits and problems, hence a comparison between both is not fair. For example, both bands can be used for many different applications such as hot-spot areas, smart cities, metropolitan and wide areas. On one hand, K/Ka-band has a larger reach than V-band, but less spectrum availability. On the other hand, V-band has a larger spectrum availability than K/ Ka-band, but higher propagation losses. 


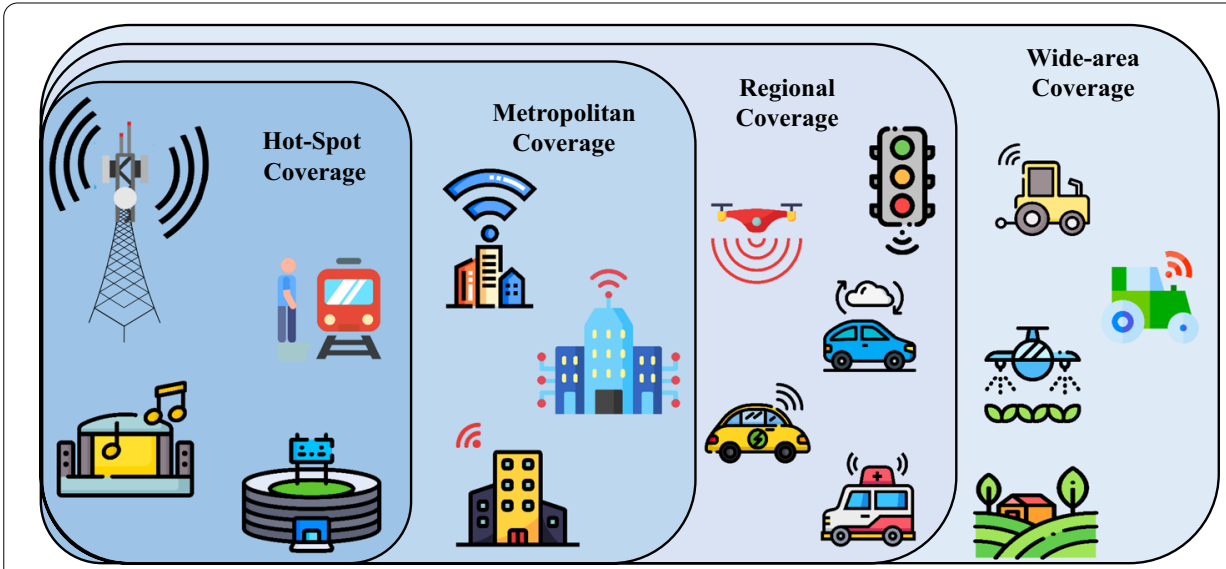

Fig. 1 Potential mmWave coverage scenarios of $5 \mathrm{G}$ mobile networks and beyond

Both ranges are lightly licensed or unlicensed bands and support considerably larger capacity than any $4 \mathrm{G}$ long-term evolution (4G LTE) standard, which renders these bands robust enough to support millions of connected devices. Therefore, both frequency regions are potential candidates for mobile fronthaul deployment in micro or small cell sites. Small cell densification and beamforming are the most promising solutions to mitigate the high path losses. Beamforming techniques concentrate the transmission and reception of the signal energy into smaller regions of space allowing space multiplexing in the wireless domain, which brings improvements in throughput, coverage, frequency re-use, and energy efficiency.

Amongst several different technologies to be potentially implemented in C-RAN fronthaul, optical space-division multiplexing (SDM), multi-core fiber (MCF), multipleinput multiple-output (MIMO) signaling [16, 17], and analog radio-over-fiber (A-RoF) $[18,19]$ stand out as key enablers. The combination of these technologies adds unique benefits, capabilities and features to a mobile network. For example, SDM can support increased data rates and several channels. SDM can support parallel independent connections between the $\mathrm{CO}$ and the remote units (RUs) in 5G networks, which enables direct implementation of both reconfigurable multiple-input multiple-output (MIMO) transmissions and remote beamforming techniques [20]. Moreover, SDM allows both digital radio-over-fiber (D-RoF) [21] and A-RoF signal transmissions to coexist in the same network. Finally, an SDM-based C-RAN can support interoperability with passive optical networks (PONs) and consequently offer a unique solution for a self-contained fiber infrastructure that can be reconfigured by software-defined networking (SDN), and network function virtualization (NFV), providing adaptive beamforming techniques, and massive MIMO.

This paper addresses the main technologies in transition that are part of 5G networks and beyond for leveraging a unique holistic mobile infrastructure. They are needed for full flexibility, interoperability, and reconfigurability. Moreover, the key-performance indicators (KPIs) of these technologies and systems are introduced and discussed. The technologies are perceived as enablers to deliver a unique end-to-end backhaul and fronthaul solution for $5 \mathrm{G}$ networks and beyond. The evolution of fronthaul mobile architectures including the concept of C-RAN is discussed. 
An experimental demonstration of a $5 \mathrm{G}$ and beyond mobile fronthaul system compliant with the 5G NR OFDM signal protocols is showcased. Results show that the system can successfully transmit 5G NR OFDM signals with both wide bandwidth (up to $800 \mathrm{MHz}$ ) and fully centralized signal processing, which renders the system capable of supporting increased capacity and consequently accommodating several simultaneous users. Finally, an experimental demonstration of a $5 \mathrm{G}$ and beyond mobile fronthaul system compliant with the 5G NR OFDM signal protocols is showcased. The system uses a set of main technologies such as $5 \mathrm{G}$ baseband unit, SDM, bespoke $10 \mathrm{~km}$ long multicore fiber with 7 cores, a pair of directional $60 \mathrm{GHz}$ antennas with $10^{\circ}$ beamwidth and SDN features. These essential enabling technologies will drive secure networks beyond $5 \mathrm{G}$ mobile systems to support millions of devices, unique quality-of-service guarantee in terms of latency, and full automation, orchestration, and management of any vertical service, use case, and applications. This paper is organized as follows. Section 2 lays out the 5G and beyond KPIs and their description. Section 3 presents the main fronthaul mobile technologies. Section 4 addresses the main backhaul mobile technologies. Discussions and experimental results are presented in Sect. 5. Finally, some concluding remarks are drawn in Sect. 6.

\section{6G key performance indicators}

$6 \mathrm{G}$ networks will provide and satisfy an extra order of magnitude in performance indicators as compared to 5G KPIs. Due to emerging technologies, unforeseen applications and use-cases, there will be a need to define a new framework and level of performance indicators along with methodologies to assess the performance of $6 \mathrm{G}$ networks in a standard and impartial way. Moreover, the use cases and services supported by $5 \mathrm{G}$ systems and beyond are ahead of traditional base station (BS) deployments but are in fact correlated with heterogeneous networking solutions that concerns a set of distinct and interoperable technologies. $6 \mathrm{G}$ networks requirements, following the roadmap of mobile technologies, will eventually provide an extra order of magnitude of KPIs. In this way, 6G KPIs will mainly support a $200 \mathrm{~Gb} / \mathrm{s}$ peak rate, density of $10 \mathrm{M}$ connections $/ \mathrm{km}^{2}$, $500 \mathrm{~EB} /$ month traffic, $300 \mathrm{GHz}$ frequency spectrum, and latency levels down to 100 us [18]. In addition, the $6 \mathrm{G}$ KPIs further will aim at user data rates reaching $1 \mathrm{~Gb} / \mathrm{s}$ for a few devices and $100 \mathrm{Mb} / \mathrm{s}$ for tens of thousands of users highly concentrated in hot-spot areas like for example football stadiums.

In addition to unforeseen applications that will emerge once the next generation mobile infrastructure is completely rolled-out, 6G KPIs will support applications such as autonomous driving systems, intelligent cooperative robotics, and autonomous lastmile delivery of goods. In most technology development processes or roadmaps, it is common to require a need at certain point that the performance of key metrics is quantified, before later steps such as prototypes and tests. To provide an accurate and unbiased assessment, the right evaluation procedures, indicators and models should be taken into consideration. Accordingly, the evaluation and target values of 6G KPIs [5] can be derived via analytical calculations, numerical simulations, or even early experiments. Indicatively, broadband access in $5 \mathrm{G}$ KPIs is expected to reach $10,000 \times$ the capacity of current 4G LTE technology, $90 \%$ reduction in power consumption, $100 \times$ better battery lifetime, ubiquitous coverage, and 10 to 100 times higher peak user data rates. Finally, 
critical levels of latency will provide a new user experience and enable unforeseen time critical applications.

6G KPIs further aim at the decrease of power consumption, which can be partially supported via PON technology for fronthaul and FSO technology for mobile backhaul. In addition, the adoption of A-RoF avoids the need for high-speed analog to digital conversion and additional modulation/demodulation stages at the remote site, which are highly demanding in terms of energy consumption. The use of SDM technology in the network is one of the most viable technologies to potentially fulfil the requirement to increase the system capacity and accomplish the target KPIs. SDM can be deployed into a network without the use of active components, hence no power is required for its operation. On top of that, the future fronthaul infrastructure will use SDN controllers that grant an overall view of the whole network, specifically useful for system management. This eventually allows for simplified and low power consumption routers and switches. Therefore, it is expected that a significant energy consumption reduction can be achieved with the novel fronthaul infrastructure.

As a matter of fact, the possible future requirements need to be defined and investigated to locate what solutions and technologies will be implemented with success. In this way, the convergence of different KPIs is of paramount importance to reflect critical success factors of a new 6G-based solution or technology. A great deal of effort has been dedicated worldwide for achieving a common sense towards convergence on 6G KPIs so as fundamental metrics could be identified and potentially standardized. There are different categories of 5G and 6G KPIs such as business, societal, and performance KPIs. They address different aspects related to a general mobile network ecosystem, from user privacy (societal KPI), global market infrastructure (business KPI), and ultra-low latency (performance KPI) [22].

Furthermore, distinct methodologies can be used to evaluate and consequently quantify them. For example, KPIs can be evaluated via an inspection method where the metrics are design-dependent and can be assessed by looking into general system design information, via analytical calculations based on the technical specifications of the system with static parameters, via numerical simulations which tackles the system modelling and evaluation by means of algorithms based on parameters that can change constantly according to the network operational conditions, and finally, KPIs can be evaluated by real network measurements in a laboratory or in the field. However, due to the complexity of networks beyond 5G and the integration of heterogeneous technologies and solutions, it might become reliable and accurate to aim at 6G KPIs by means of experimental investigations and measurements. Despite all worldwide efforts dedicated on the elaboration and evaluation method of $6 \mathrm{G} \mathrm{KPIs,} \mathrm{it} \mathrm{is} \mathrm{worth} \mathrm{pointing} \mathrm{out} \mathrm{that} \mathrm{the}$ definition of KPIs and methodologies of evaluation is an ongoing work and effort.

KPIs, from a practical perspective, are supposed to be measured and validated during both laboratory and field trial tests. In this fashion, to perceive a common plan towards convergence of $5 \mathrm{G}$ and beyond KPIs such as latency, peak data rate, and energy efficiency, has become crucial for next generation mobile systems. Even if KPIs might be different from source to source, mainly due to specific technologies, they still need to converge. Moreover, KPIs should consider different usage scenarios or use cases with regards to distinct markets (automotive, eHealth, media and entertainment), each of 
them with a distinct performance requirement to support different needs. Normally, these use cases are addressed in a way that they fit one of the following categories: enhanced mobile broadband (eMBB) use case, ultra-reliable low latency communications (URLLC) with a highly heterogeneous pool of resources to support different application requirements and services, and massive machine-type communications (mMTC) use case.

\section{6G fronthaul technologies}

In general, a C-RAN fronthaul architecture regards the relocation of the baseband units (BBUs) from the remote RU (also known as remote radio head) premises to the CO site creating a centralized pool of BBUs as illustrated in Fig. 2. Such a paradigm can grant the mobile network several advantages such as higher flexibility and manageability, reduced power consumption, along with the addition of virtualization and softwarization features. Traditionally, radio heads (now referred to as RU) and BBUs were physically located at the same cell tower with a coaxial wire connection from the top of the tower (RUs) to the bottom of the tower (BBUs). Once this link was replaced by optical fiber, the BBUs sitting at each tower were moved deeper into the network and placed in a centralized and distant location, and this new connection between the now remote RUs and stacked centralized BBUs (also referred to as single cloud-BBU) defines the fronthaul mobile network.

A RoF fronthaul networks [18] transmit a baseband signal throughout a fiber path, undergoes optical to electrical conversion $(\mathrm{O} / \mathrm{E})$, digital to analog conversion and upconversion to the radio frequency, and then is radiated and detected. Thereby, the $\mathrm{O} / \mathrm{E}$ conversion represents the transition from the fiber link to a radio frequency signal at mmWave frequencies, which denotes the main step in bringing advantages to RoF-based networks. The use of analog RoF with the radio frequency signal already generated at the $\mathrm{CO}$ enables a network to employ larger modulation bandwidths available in the optical domain, and further enables with ease the centralization of critical equipment such as network control, and radio frequency oscillators. Hence, an analog RoF fronthaul system can benefit from the wireless and optical domains in a way that fiber optics bring the ability to easily bridge large distances and use very large modulation bandwidths, while wireless links allow for easy, fast and flexible deployment, and additional simplification of centralized networks, regardless of the multiplexing technology deployed.

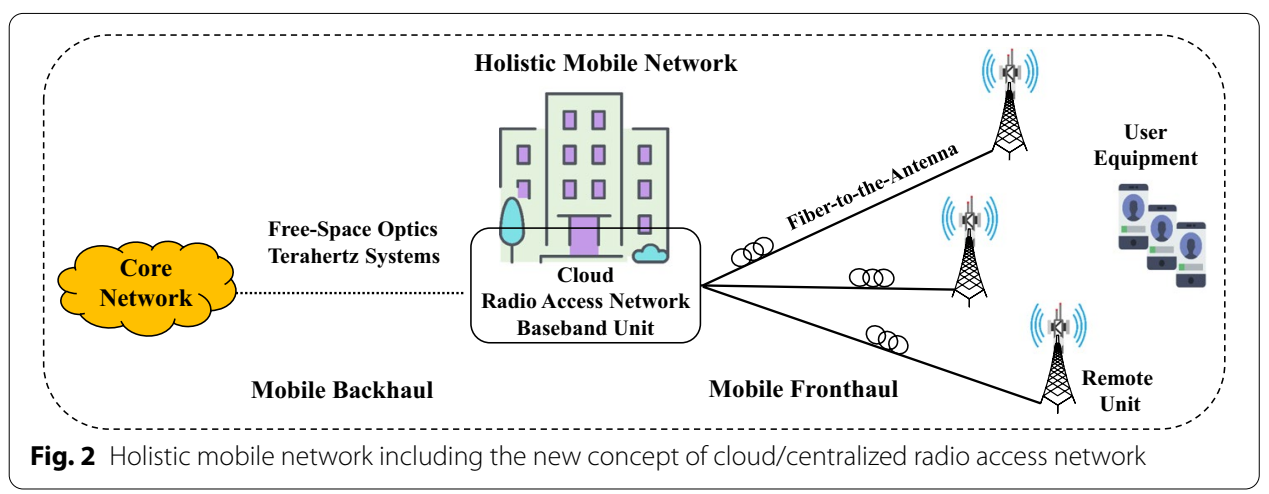


Ultra-dense WDM multiplexing technology along with ROADMs based on photonic integrated circuits can be used in the fiber path so that intelligent and reconfigurable functionalities can be implemented while keeping compatibility with legacy backhaul and fronthaul infrastructure. The combination of both technologies can bring the network capacity to another extent, supporting, for example, ultra-dense scenarios like the 2020 European football championship event. Accordingly, such an event can gather a vast number of people interested in watching and transmitting super high-resolution video streaming, which generates an immense amount of traffic data requiring tremendous network capacity. In addition, end users can upload multimedia content inside and surround stadium premises [23]. As a result, peaks of data traffic happen due to the large number of simultaneous users gathered in the same place as well as during the same time interval. Thus, only heterogeneous technologies leveraged together by means of softwarization including virtualization can accommodate thousands of hundreds of simultaneous users and satisfy such a challenging networking scenario.

Alternatively, in a long term deployment scenario, SDM technology in the optical domain in ribbon or multi-core fibers (MCFs) [24] is regarded as a major technology player in increasing fronthaul network capacity, peak data rates, and flexibility for supporting such an unprecedented and insatiable demand of data traffic requested not only by football events, but also Olympic games, musical concerts, and large commemorative events. Another technology worth attention is ribbon optical fibers that use a single loose tube with rollable ribbon fibers as an alternative solution for connecting several individual fibers from the $\mathrm{CO}$ premises to the 5G RUs for providing greater network capacity and broadband transmissions. The reduced physical footprint from denser fiber techniques such as the rollable ribbon fibers are perceived as a prospective alternative solution for deploying fronthaul mobile infrastructure in a way that it can satisfy the stringent capacity requirements not only of $5 \mathrm{G}$ mobile systems, but also of coming $6 \mathrm{G}$ systems.

Noteworthy, ribbon fibers have a smaller diameter and weight and can provide a higher density as they are rollable into a smaller package than conventional flat ribbon fibers, with each of their individual fiber ease to be manipulated. Finally, ribbon fibers might be the transition technology to drive current systems towards a 6G fronthaul mobile network, where not only is considerably footprint reduced, but also the channel conditions observed by signals propagating in different cores are more robust than those for signals propagating in different fibers of a ribbon. In this fashion, MCF remains needed and ideally more suited for advanced combined optical and RF technologies such as optical beamforming. In addition to added capacity, reduced footprint and more uniform channels, MCF may also allow for joint signal treatment across multiple cores, when considering combined SDM and WDM transmission or the possibility of joint amplification across all cores, leading to further flexibility and potential reductions in energy consumption.

Furthermore, SDM along with MCF technology can not only support increased capacity of 6G mobile systems in a cost-effective way, but also be compatible and use current deployed fiber technology towards a seamless transitional solution. This combination of MCF and SDM technologies can allow a massive deployment of RU in an A-RoF fronthaul network and consequently meet challenging 5G KPIs as well as dense use 
case scenarios like the already mentioned 2020 European football championship event. Finally, these technologies further enable the use of beamforming techniques allowing enhanced control over the spatial signal distribution, which significantly increases the user data rate with a minor added cost at the remote RU side.

By its turn, the beamforming technology can be achieved in both RF domain along with microelectronics by using true time delays or phase shifting, and in the optical domain along with photonic integrated technology by employing similar adapted techniques on a single chip but with most likely enhanced overall performance. A third and distinct way to implement beamforming solutions is based on a hybrid beamforming system and approach that combines both RF and optical methods. Solutions based on beamforming rely on improved efficiency by today's manufacturing techniques of complex circuits with reduced size, weight and energy footprint. The large difference in wavelength between optical and RF signals, allows the use of photonics for implementation of complex beamforming networks, alleviating the challenges faced by traditional RF beamformers in terms of footprint energy usage and heat dissipation. Interestingly, both blueSPACE and 5G-PHOS projects focus on the concept of optical beamforming, however in complementary fashions, with 5G-PHOS targeting larger antenna arrays with a single, highly focused beam, whereas blueSPACE focuses on the natural transmission of multiple independent beams from a single antenna array.

\subsection{G network softwarization}

A key field for developing the future virtual networking architectures of $6 \mathrm{G}$ systems is softwarization solutions that are not based on traditional pure hardware solutions. 6G systems will feature a full range of software-based functionalities powered by agile SDN, NFV, and fog computing capabilities [25-32]. In addition, network softwarization embraces zero-touch service management, automation, security, and trust networking features, to name a few. The cloud RAN [26], where baseband functions are moved deeper into the network in more centralized locations as compared to legacy architecture, has support for multi-tenancy network sharing and slicing. The combination of all these network features should provide ideal flexibility and re-configuration of the system in a way that any sort of service can be fully satisfied. Softwarization further allows for decoupling many network functionalities from the underlying hardware with them running purely in software by using virtual machines in a seamless manner.

Finally, the augmentation of existing SDN concepts and implementation of NFV functionality with the novel network structures and ARoF transmission paradigms in mind is a key element. The implementation of targeted SDN and NFV frameworks for ARoF transmission over both WDM and SDM fronthaul networks is considered in the projects and will be a key contribution to the value and operability of the developed fronthaul solutions as well as to achieving the 5G KPIs on capacity and service deployment time. By employing SDN-aspects over A-RoF transmissions allows controlling not only the wavelength of the optical carrier, but also the intermediate frequency over fiber at which the analog streams are transmitted, introducing enhanced granularity in subcarrier multiplexed A-RoF transmission [33]. Moreover, virtual functions can create not only virtual instances of hardware platforms, but also operating systems, storage devices, and most computing resources. The NFV can be used in both cloud and fog computing systems, 
providing on-demand access to different applications and services by sharing a flexible pool of computing resources [34-36].

The mobile edge computing (MEC) node concerns a group of functionalities and technologies achieved via AI, SDN, NFV, slicing, service orchestrator, and traffic classification. SDN can resolve the vertical integration of current network environments by decoupling the control plane (i.e., control logic) from the data plane (i.e., data forwarding equipment). With such an architecture, routers and switches become simple forwarding network elements whose control logic is provided by an external entity called SDN controller or network operating system (NOS). Northbound interfaces (NBIs) offered by a logically centralized SDN controller allow different network applications (firewalls, routing, and resource orchestrators) to implement network control and operation logic. In addition, other type of high-level NBIs category are implemented as NOS management applications. Examples of this category include virtual tenant networks, application-layer traffic optimization, and intent-based networking (IBN). In SDN, the concept of orchestration is vital to automate network operations properly. SDN network domains need single-domain or multi-domain orchestration systems to coordinate endto-end connectivity services through different network domains controlled by different SDN controller instances, which in turn must communicate directly with the physical network. Furthermore, the leverage of AI and SDN capabilities can give rise to a system framework in which software and hardware from different vendors can be used with ease and flexibility, instead of being limited to a specific commercial and proprietary solution. To put it simply, the AI system of the SDN-orientated solution considered here is transparent for the network hardware components and can be run remotely in the MEC node. The SDN networking paradigm along with $\mathrm{AI}$ is leveraged to have control over the data flow [27]. On top of that, both SDN controller connections to the 5G towers can be achieved by leveraging the $5 \mathrm{G}$ systems that by its turn enables network topology change on demand, consequently adapting to the changing needs of different conditions, traffic, and urban environments. By its turn, network service orchestration has become a key feature to enable the delivery of different 5G KPIs. A framework orchestrator focuses on the support of VNF operation across different hypervisors and computing resources. It also covers the orchestration and lifecycle management of physical and virtual resources. In the NFV context, the orchestrator can have two main functions including resources orchestration across multiple VIMs and network service orchestration.

Network slicing functionalities will consider available spectrum, computational and storage resources, sensor gateways, small cells, and fog servers, to enable the appropriate placement of VNF, security, service orchestration, and traffic prioritization. The BBU functionalities include virtualized RAN (vRAN) to fully virtualize the 5G infrastructure elements. This approach will seamlessly interface the interacting facility user with the entire underlying 5G RAN environment, ensuring that (a) a diversity of wireless communication technologies can be served, and (b) edge network logic can be utilized for dynamic and de-centralized service deployment. Its capability to accommodate in a dynamic manner, user service logic on the edge network comes to fulfil the requirement for network resilience and transparency, so that network agnostic services can be provided either locally on specific network branches or at a global level. 


\subsection{Beyond massive MIMO systems}

MIMO technology allows the transmitting and receiving of more than one signal simultaneously over the same radio channel [30]. MIMO systems can multiply the capacity of a wireless connection without requiring more spectrum with considerable capacity and throughput improvements and could potentially yield as much as a 50-fold increase in next generation mobile networks. In fact, massive MIMO technology is a key enabler and foundational component of those networks.

Mobile fronthaul systems will rely not only on beamforming and beamsteering technology [37], but also on massive MIMO technology for creating several transmission channels at mmWave band. Massive MIMO uses several antennas arrays to provide signal amplification by beamforming and high spatial resolution to multiplex many simultaneous users. MIMO systems are based on phased array antenna devices with thousands of antenna elements. The radio interface, which combines mmWave carriers with MIMO as well as beamforming and steering capabilities, maximizes the capacity and ensures signal quality regardless of user location and motion. The previous generations of mobile networks the use of massive antenna arrays was considered impractical due to form factor and size restrictions, but with the introduction of mmWave signals many antenna elements can fit within a small area. This allowed moving from small MIMO implementations with a few antennas only towards massive MIMO with many antennas. The main concept is to equip base stations with arrays of many antennas, which are used to serve many terminals simultaneously, in the same time-frequency resource. Moreover, new frequency and time division duplex protocols and more advanced algorithms should be developed for massive MIMO systems. MIMO algorithms play a critical role to control how data maps into antennas and where to focus energy in space [38]. Whilst massive MIMO is currently most compelling at sub-6 GHz region, several developments aim at MIMO implementations at the mmWave frequency band.

Beyond massive MIMO expands the massive systems by employing an even much larger number of antennas elements. Beyond massive MIMO technology based on novel antenna architecture will potentially use smaller area and loads of antenna elements for multiple wideband beam transmissions and consequently will achieve a considerable larger capacity as well as area coverage with substantial spectrum efficiency and power consumption reduction. Accordingly, beyond massive MIMO systems featuring an unprecedent number of beams transmissions will be capable of accommodating several simultaneous users highly located in the same region such as hot-spot areas. Massive MIMO's feature to support multiple simultaneous users within a condensed area while keeping high-speed data rates and consistent performance makes it the perfect technology to address the needs of 5G and beyond networks. Eventually, the antennas of such a MIMO system will have a unique architecture based on PIC technology with antenna arrays having very small form factors to meet new requirements. The goal is to have antennas help by focusing energy into ever smaller regions of space to bring large improvements in throughput and radiated energy efficiency pushing severalgigabit transmissions over a system. As a conclusion, regardless of the frequency range employed, massive MIMO technology will be part of next generation networks as an efficient solution for supporting higher data rates, spectral efficiency, and capacity. 


\subsection{Photonic integrated circuit technology}

Current advances in mobile systems endeavor to find solutions that can meet requirements not only of today's networking scenarios, but also future requirements not yet unveiled. Part of today's challenges in mobile systems is related to scalability, processing capability, and energy consumption of optical wireless solutions. It is very challenging and unrealistic for a solution to cope with and be able to satisfy all requirements via a single technology. Nonetheless, the combination of optoelectronics, photonics, and microwave as well as mmWave techniques can be a paramount technological alternative for solving challenging problems [39-41]. Photonic technology is immune to electromagnetic interference, has both great accuracies to control independently phase and amplitude and fully programmable capability with wideband high-speed signal processing functionalities. This field has attracted a great deal of attention with recent trends being directed towards photonic integration [41]. The recent progress in PIC technology will potentially lead to a wide variety of economically feasible $6 \mathrm{G}$ mobile and fronthaul and backhaul devices capable of handling and processing an unprecedented volume of data traffic with low energy-consumption as well as secure- and cost-efficiency, creating new alternative solutions. In addition, PIC technology can long-term reduce the cost of and enable massive fabrication of devices and have smaller footprint, enabling flexile device architectures and designs and consequently more robust and powerful systems.

Photonic processors have inherent speed capabilities to manipulate signal processing functions with high resolution and wideband. Moreover, they provide the ability to perform parallel signal processing, and the ability to generate true time delays, that can be used as an alternative solution and open new opportunities in $5 \mathrm{G}$ networks and beyond. For example, this can be used in advanced phased array antennas with thousands of antenna elements, in UDWDM-based ROADM and filter devices, random-number generators, and quantum-key distribution devices. Another concrete example of photonics signal processing is its use along with fiber Bragg gratings to resolve individual frequency bands and create differential true time delay among them to perform signal processing functionalities such as classical logical circuits in a photonic way for a vast range of applications in optical wireless systems [40]. Such a photonic-based solution can add many benefits to a system such as compactness as compared to traditional solutions, performance stability against mechanical conditions, and size as well as weight reduction. Photonic signal processing can handle multi-GHz sampling frequencies overcoming microelectronic devices' limitations. The former is compatible with classical optical fiber and free-space systems and can support built-in signal conditioning for performing parallel signal processing. Photonic signal processing can be further used in photonic analog-to-digital converters, arbitrary waveform generators, and frequency measurement subsystems. A different configuration and design are required for different applications and solutions, but the principle remains the same. Thus, PIC-based devices along with photonics signal processing will eventually be key technologies of several solutions to be implemented at different parts and stages of the holistic optical wireless next generation networks. 


\section{6G backhaul technologies}

Mobile backhaul normally refers to the part of the network that connects the BBU located in cell sites to the mobile core. Mobile backhaul is becoming even more important in 5G networks and beyond since the latter has larger densification of site cells with thousands of user equipment and unprecedented data traffic demand. By its turn, the capacity requirement of transport networks increase considerably as the fronthaul network evolves. Mobile backhaul concerns a transport network that connects the core network and the C-RAN of an end-to-end mobile system. Accordingly, mobile backhaul is responsible for conveying the data traffic of user equipment between the C-RAN central office and the core network. Among many different technologies considered for the backhaul infrastructure, wireless FSO and THz systems are considered prospective candidates as alternative to fiber optics due to their unique available capacity. Moreover, point-to-point FSO and THz systems can cope with challenging requirements such as latency and reach. These interesting technologies can be part of key solutions for mobile backhaul infrastructure as an alternative to leased wired fiber-based solutions.

\subsection{Terahertz systems}

Communication systems in the $\mathrm{THz}$ frequency range have received increased attention recently fostering several new possibilities and applications [42-44]. THz systems are enablers of inter- and intra-vehicle communication in a way that vehicles can communicate with one another, traffic lights, and provide intra-connectivity to devices and components in the inner part of the vehicle. The latter, enabled by $\mathrm{THz}$ wireless systems, can build a wireless personal area network inside a vehicle supporting high-speed transmission rates. THz wireless systems can be considered a complementary solution to work along with $5 \mathrm{G}$ and beyond networks. These technologies complement each other in many respects and should be integrated according to network requirements. With autonomous driving vehicles becoming a sort of mobile data center due to the large amount of traffic they are expected to handle in the soon future, in order the of peta-bytes, THz systems have become a key technology to satisfy real-time traffic demand in heterogeneous networks by easing the spectrum scarcity and capacity limitations of current wireless systems [43].

Accordingly, THz wireless systems can offer a large enough bandwidth and satisfy this new data increasing trend in autonomous vehicles. To do so, new techniques for signal generation, wireless transmission, and data recovery have been investigated [45]. The frequency range between $300 \mathrm{GHz}$ and $3 \mathrm{THz}$ offers huge bandwidths of several tens of $\mathrm{GHz}$, and as an example, the first standard for $\mathrm{THz}$ communication (IEEE 802.15.3d) considers transmission rate up to $100 \mathrm{~Gb} / \mathrm{s}$. Although obstacles like trees, poles, and concrete fences can block $\mathrm{THz}$ signals, autonomous relaying technologies can be used to remedy these issues. For example, vehicle relays can be used to create an ad hoc network among several autonomous vehicles so that they can bypass obstacles and keep the communication system functional. Nonetheless, $\mathrm{THz}$ systems can surprisingly support up to $100 \mathrm{~m}$ links with directional antennas and up to $10 \mathrm{~m}$ with omnidirectional antenna [42]. The frequency range of the different wireless technologies can be found in [45]. 
Recently, progress in semiconductor technology has made communication systems beyond $275 \mathrm{GHz}$ possible. The $\mathrm{THz}$ frequency range between $300 \mathrm{GHz}$ and $3 \mathrm{THz}$ offers huge bandwidths of several tens of $\mathrm{GHz}$ if the so-called passive services like earth observation and radio astronomy are not disturbed by interference. Currently the wireless backhaul solutions planned for $5 \mathrm{G}$ achieve only up to $10 \mathrm{~Gb} / \mathrm{s}$. THz radio link, the ThoR project realizes for the first time both the enormously high real-time transfer rates up to $100 \mathrm{~Gb} / \mathrm{s}$ and the implementation of the new IEEE standard for a backhaul or fronthaul system. Further solutions based on THz technology aim at MIMO systems for $\mathrm{THz}$ communications to achieving up to $1000 \mathrm{~Tb} / \mathrm{s}$ transmissions.

\subsection{Free-space optical systems}

FSO is a line-of-sight technology that uses a light beam from semiconductor lasers for data transmission instead of wired optical fibers or wireless micro/mmWave RF links. Compared to optical fiber cables, FSO systems transmit light through the air medium or free-space instead of a fiber [46]. FSO technology has an extensive list of benefits such as vast available spectrum, unlicensed spectrum and ease deployment [47]. Moreover, FSO is considered a flexible and scalable technology that might be implemented in a costeffective way as compared to traditional backhaul solutions. Furthermore, FSO technology is regarded as a promising solution for mobile backhaul due to the densification of site cells and consequently shorten distance among them and C-RAN, reduced to a few hundred meters. In addition to its similar capacity, FSO is more cost-efficient than optical fibers for regions with poor or minor fiber infrastructure in the way that FSO systems allow easy installation and uninstallation for creating new communication links within remote areas with short installation time. Finally, FSO is immune to any electromagnetic interference that can be generated by surrounding RF devices.

As a potential solution for backhaul mobile of next generation mobile networks, FSO should satisfy key networking requirements and consequently support high data rates. Several works have been dedicated on the progress of FSO systems [48-55]. Several FSO-based solutions can achieve up to hundreds of $\mathrm{Gb} / \mathrm{s}$ and beyond transmissions. For example, an FSO system capable of achieving transmissions up to $\mathrm{Tb} / \mathrm{s}$ via a wavelength sharing technique was proposed in [52]. In addition, an FSO prototype that can reach up to $100 \mathrm{~Tb} / \mathrm{s}$ was demonstrated in [55].

Another FSO system supporting up to $1.28 \mathrm{~Tb} / \mathrm{s}$ with $32 \times 40 \mathrm{~Gb} / \mathrm{s}$ WDM channels under tests running during an entire day with stable transmissions including minor bit error rate (BER) fluctuations was demonstrated in [56]. Also, a hybrid fiber FSO system without optical-electronic-optical conversion at the interfaces with air capable of achieving transmissions up to $1.6 \mathrm{~Tb} / \mathrm{s}(16 \times 100 \mathrm{~Gb} / \mathrm{s})$ was reported in [57] . Alternatively, a hybrid network that uses both mmWave and FSO technologies was proposed in [58] as a potential complementary solution for classical mobile backhaul networks. Recently, a new hybrid network based on the seamless combination and leverage of FSO and RoF technologies for mobile fronthaul have been experimentally demonstrated [59-61]. This network can provide broadband coverage to remote rural areas due to its capability of link extension based on the FSO technology. Interestingly, the network accounts for mmWave signal generation based on a photonic-assisted all-optical up-conversion technique [60]. 
In addition to constraints like interference due to ambient light, scattering and physical obstructions on the path of FSO links, both atmospheric turbulence-induced fading also known as scintillation and strong path-loss constitute the major impairments associated to FSO links limiting their performance and reach. Even though FSO systems suffer from different atmospheric impairments (i.e., fog, scintillation), several works have addressed new techniques to mitigate these problems. For example, a new method based on deep learning for atmospheric impairment compensation of FSO systems has been proposed in [62]. Interestingly, the latter improves the BER of the system by applying supervised training techniques to compensate the phase screen in randomly changed turbulent environments. A second solution for mitigating atmospheric impairments is a scheme based on generative machine learning that corrects distortion effects and reduce detection noises in the FSO link [63]. This method results in a significantly lowered symbol error ratio and crosstalk at the output of the receiver while requiring no additional complex signal feedback. Finally, many traditional techniques based on diversity have been proposed for the mitigation of atmospheric issues in FSO systems [64]. Regardless of the technique, many of these impairments might be drastically reduced in next generation mobile networks with the help of AI and machine learning techniques along with PIC technology. The latter can bring many benefits to FSO links like substantial performance improvement by means of channel crosstalk reduction based on a new design of transceivers. Hence, FSO systems are a strong candidate to meet 6G KPIs and pose the technology in an enough maturity level to be implemented in 5G and beyond mobile backhaul networks.

\section{Results and discussion}

In this section, a preliminary demonstration for emerging technologies for systems beyond 5G developed by a collaboration between blueSPACE [65] and 5G-PHOS [66] projects is presented. The joint demonstration shows the potential of analog techniques for mobile fronthaul as a viable alternative for next generation mobile systems. Indeed, the experimental demonstration aims to point out analog mobile fronthaul as a key candidate to serve future mm-wave $5 \mathrm{G}$ and beyond systems by showcasing the feasibility of analog transport of large bandwidth radio signals within the mm-wave frequency band, compliant with 5G NR protocol in fully centralized signal generation and processing as part of a series of developments of the two projects. The demonstration setup features a combined optical wireless downlink system based on I\&Q over fiber (IQoF) transmissions with a simplified schematic shown in Fig. 3.

The setup has a BBU with SDN functionalities like slicing for 5G RoF mobile systems, a $10 \mathrm{~km}$ long prototype MCF with 7 cores and a pair of directional V-band antennas. Furthermore, the usual baseband processing functions as well as analog to digital

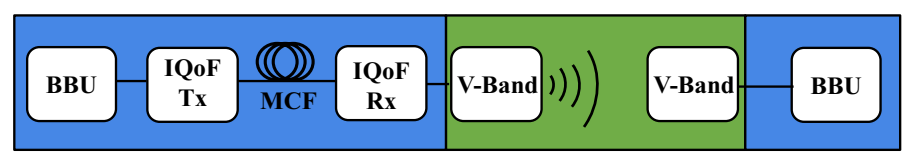

Fig. 3 Proposed schematic for the mmWave point-to-point link for the joint demonstration of blueSPACE and $5 \mathrm{G}-\mathrm{PHOS}$ 
conversion are performed remotely at the $\mathrm{CO}$ by the ARoF BBU developed by blueSPACE. This centralization enables a simplification of the RU to a basic optical Rx and antenna module, which operate at $60 \mathrm{GHz}$ (V-band) and was developed by 5G-PHOS project. The optical link supports SDM with MCF channels; more specifically, optical in-phase and quadrature $(I \& Q)$ signals are transmitted over different cores of the same MCF prototype. The experimental setup of the 5G IQoF system is shown in Fig. 4. The same ARoF BBU is used for both $\mathrm{CO}$ transmitter and user receiver in a close loop for the sake of simplicity.

The setup counts on at least the following technologies: (a) ARoF BBU with SDN functionalities that consists in four boards: filed-programmable gate array (FPGA), digitalto-analog (DAC), analog-to-digital (ADC) and clock (CLK). The FPGA is programed to (de)modulate 5G NR signals, including the digital signal processing (DSP) functions, in real time. Both DAC and ADC have single ended I\&Q outputs/inputs, sample rates above 1 Gbps. The CLK board includes a phase-locked loop (PLL) and it provides the reference clock of $1 \mathrm{GHz}$ to DAC and ADC. (b) A MCF prototype that allows that both signals are transported over the same fiber, even though they are at the same wavelength. The MCF used in this setup has 7 cores, a length of $10 \mathrm{~km}$ and a custom fan-in (FI) and fan-out (FO) adaptors designed by the blueSPACE project. The MCF was characterized and has a maximum insertion loss (IL) around $4.63 \mathrm{~dB}$ between the cores and a polarization dependent loss (PDL) up to $0.11 \mathrm{~dB}$. And (c) directional $60 \mathrm{GHz}$ antennas. The 5G-PHOS V-band antenna modules feature $9 \mathrm{GHz}$ tuning range centered at $60 \mathrm{GHz}$, larger to $400 \mathrm{MHz}$ signal envelope and $36 \mathrm{dBi}$ gain, and a $10^{\circ}$ beamwidth. The Tx module upconverts the baseband I\&Q signals to V-band, while it also integrates a V-band power amplifier (PA) and local oscillator in a fully packaged antenna, that employs differential $I \bar{I}$ and $Q / Q^{-}$input configuration with average RF power up to $+1 \mathrm{dBm}$. Similarly, the $\mathrm{Rx}$ module features a similar configuration, to down-convert the received signals. The $\mathrm{Rx}$ antenna board includes a V-band low noise amplifier (LNA) and a dedicated integrated frequency reference clock on board. Thus, the main custom-made devices of the setup are described. The setup also counts with commercially available devices such as lasers, Mach-Zehnder modulator (MZM), amplifiers and a photodiode (PD). Due to physical space limitation during the measurements, the distance between the two antenna modules was set to $4 \mathrm{~m}$, which is below the minimum design distance and may cause PA and LNA saturation.

Accordingly, I\&Q signals are transmitted through two separate optical channels at the same wavelength to avoid the introduction of excessive skew between the I\&Q signal

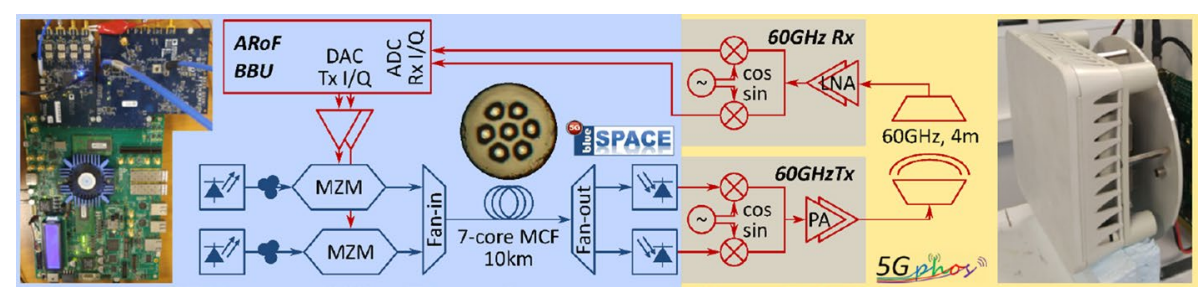

Fig. 4 Experimental setup of the mmWave link of the joint demo. Featuring the key technologies: ARoF BBU, MCF and $\mathrm{V}$-band antenna modules 

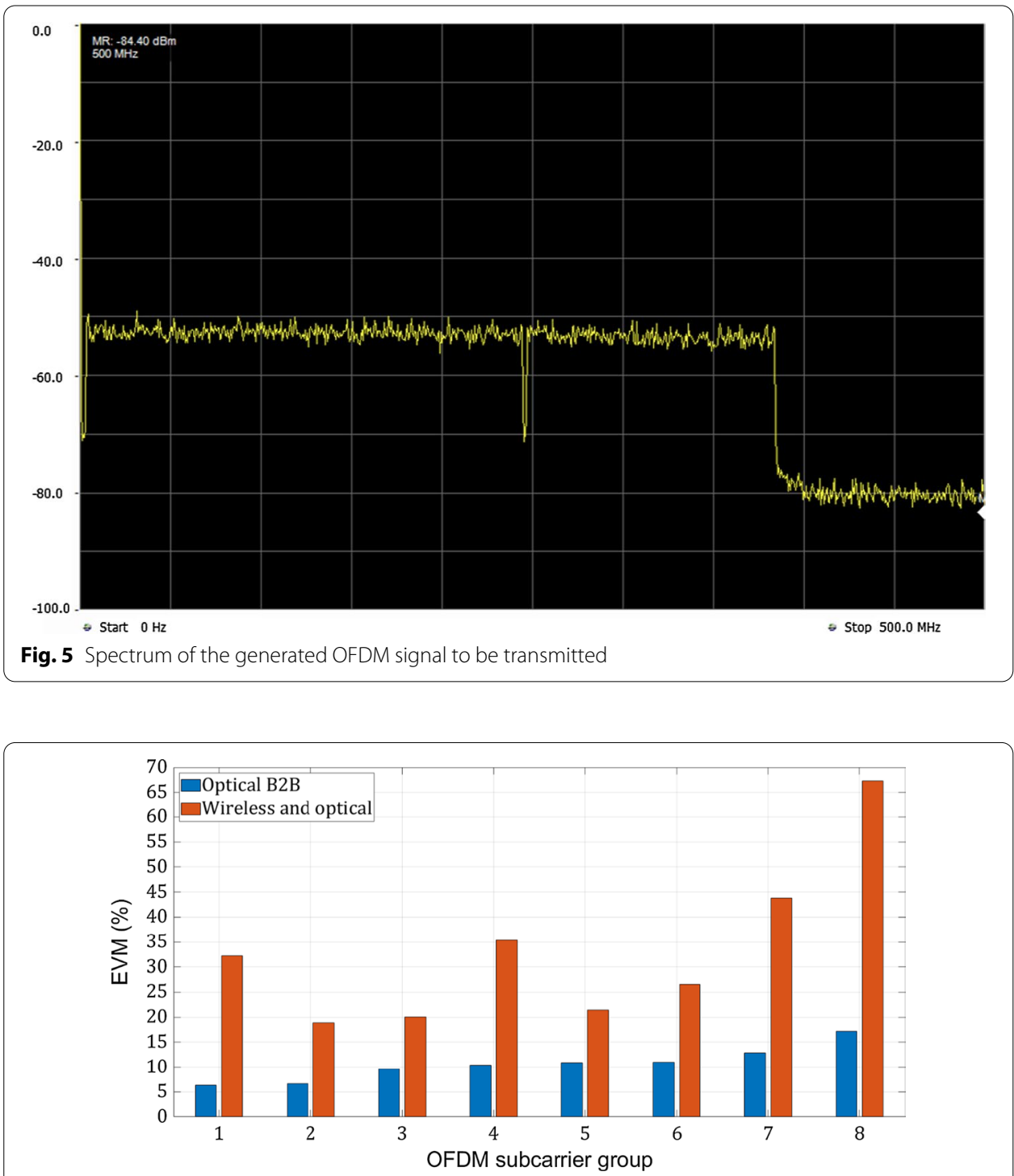

Fig. 6 Error vector magnitude as a function of different OFDM groups

components in the $\mathrm{C}$ band with the two channels including COTS; more specifically a laser, MZM with driver amplifier and a PD for each channel. The BBU transmits pseudo random bit sequence (PRBS) data at up to $1.5 \mathrm{~Gb} / \mathrm{s}$ and (de)modulates the same to a $5 \mathrm{G}$ NR compliant OFDM signal with a total of 4096 subcarriers spaced at $240 \mathrm{kHz}$, of which 3136 are active, resulting in an effective signal bandwidth up to $800 \mathrm{MHz}$. The modulation applied to these subcarriers is quadrature phase-shift keying (QPSK). Additionally, the $\mathrm{BBU}$ includes a digital to analog converter (DAC) and analog to digital converter (ADC) generating and receiving separate I\&Q baseband signals, respectively. Figure 5 shows the spectrum of one of the baseband I\&Q components at the transmitter output of the 5G BBU; notice that the spectrum of the signal occupies nearly $400 \mathrm{MHz}$, leading to a combined IQ bandwidth of $800 \mathrm{MHz}$. 


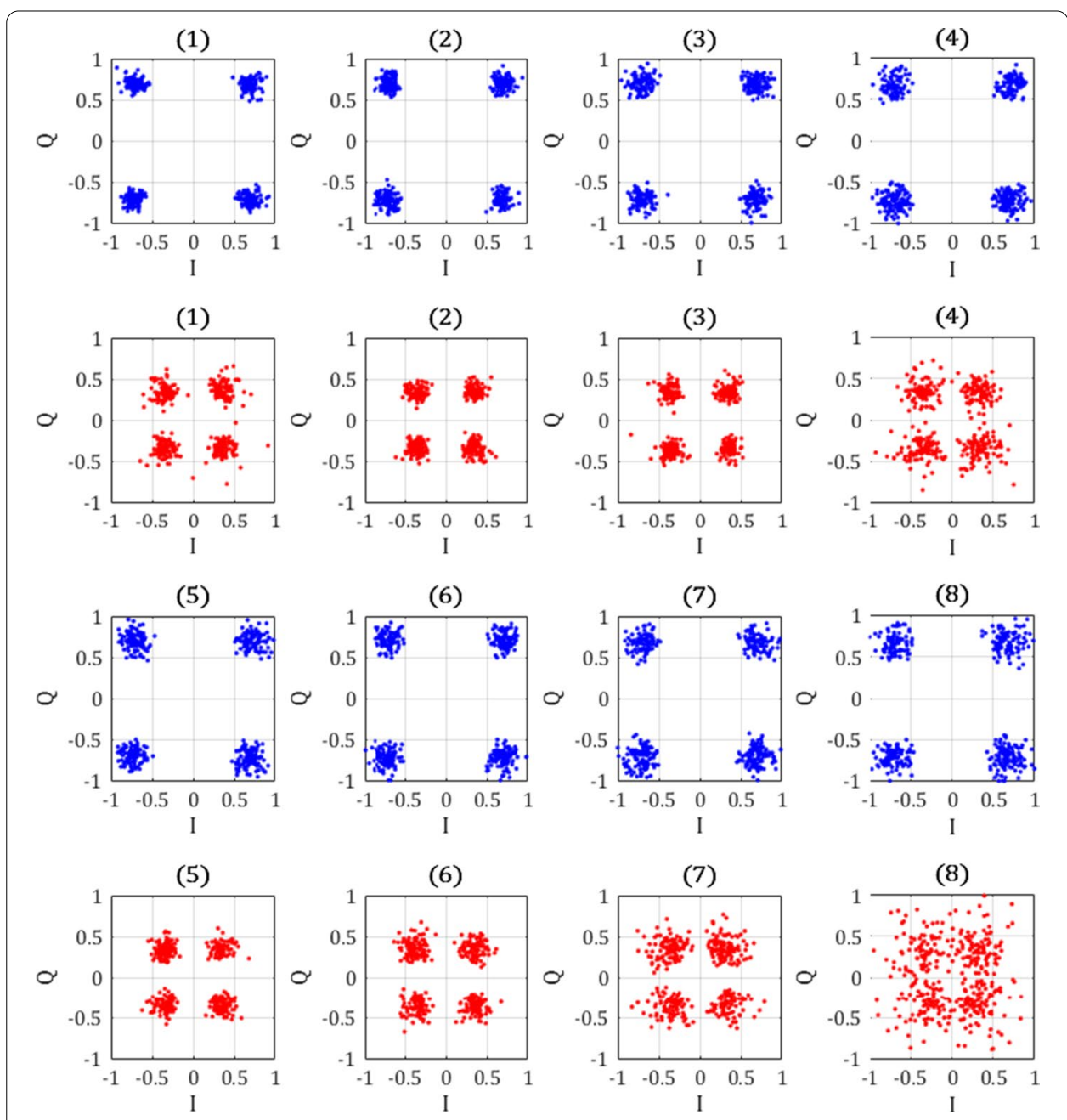

Fig. 7 Constellation diagrams of the received QPSK signal per OFDM group. Row 1 and 3: optical B2B; row 2 and 4: after wireless transmission

Next, Fig. 6. shows the measured error vector magnitude (EVM) of the transmitted QPSK signals at the different OFDM subcarrier groups whereas their respective constellation diagrams as shown in Fig. 7. The gains of the V-band amplifiers and BBU have been optimized to achieve a tradeoff between signal-to-noise ratio (SNR) and saturation. Experimental results include transmissions with optical back-to-back (B2B) including the MCF but without wireless, i.e., connecting the PDs to the BBU, and with the complete IQoF link including mmWave wireless transmission. For easy visualization and guidance, the subcarriers (SC) of the OFDM signal are split in eight groups of equal size, each covering ca. $100 \mathrm{MHz}$ of signal bandwidth (it should be noted that this is done purely for visualization and signal processing is performed on all subcarriers simultaneously). Optical B2B results show EVM below $20 \%$ for all the SC groups with the best performance achieved at the lowest frequencies, i.e., at $\mathrm{SC}$ groups 1 and 2. The maximum SNR is $23.9 \mathrm{~dB}$ at group 1 , while the minimum is $15.2 \mathrm{~dB}$ at the highest frequency, i.e. SC group 8 . When introducing the wireless link, groups 7 and 8 are strongly affected by the bandwidth limitations of the V-band modules, while group 4 suffers from degradations 
due to non-flat gain/channel response. Up to SC group 6 the IQoF link achieves EVM below $30 \%$ except for the SC groups 1 and 4 . In this case the maximum SNR is $14 \mathrm{~dB}$ at $\mathrm{SC}$ group 3, while the minimum SNR is $3.4 \mathrm{~dB}$ at $\mathrm{SC}$ group 8. The EVM deterioration when adding the wireless channel, is mainly due to the limitations of the V-band modules in terms of bandwidth and the available physical distance between the antennas. More specifically, the V-band modules are designed to operate above $10 \mathrm{~m}$ distance; therefore, both the amplifier gains at the transmitter and receiver were reduced to their minimum settings to avoid saturation. Nonetheless, noise performance of the amplifiers is expected to be suboptimal at this setting and some signal compression is experienced.

As a result, the preliminary results of the joint setup demonstration proved with success that $5 \mathrm{G}$ mmWave ARoF systems can be a prospective candidate to drive highcapacity networks beyond $5 \mathrm{G}$.

\section{Conclusions}

This paper tackled the main technology enablers in the transition towards $6 \mathrm{G}$ mobile networks. The key functionalities of the technologies were introduced and discussed. The continuous hardware and software solutions convergence is creating new possibilities for the core and fronthaul network to be closer than ever along with baseband unit functionalities being heavily migrated to the cloud via softwarization, virtualization, and AI, it is clearly an exciting future direction with novel solutions to be exploited. The coming years will eventually witness novel efforts based on a full software implementation. Among a vast set of heterogenous technologies, beamforming, FSO and THz systems, multi-core fibers, and massive MIMO transmissions, along with virtualization and softwarization were addressed and beneficially exposed to be exploited in 6G systems. The emerging technological development to drive towards secure $6 \mathrm{G}$ systems will rely mainly on the seamless interoperability of heterogeneous solutions.

An experimental demonstration of a $5 \mathrm{G}$ and beyond mobile fronthaul system compliant with the 5G NR OFDM signal protocols is showcased. Results show that the system can successfully transmit 5G NR OFDM signals with both wide bandwidth (up to $800 \mathrm{MHz}$ ) and fully centralized signal processing, which renders the system capable of supporting increased capacity and consequently accommodating several simultaneous users. Finally, this preliminary experimental result validates the potential of these technologies to satisfy $6 \mathrm{G}$ KPIs, challenging networking scenarios, and to clarify the transitional path until $6 \mathrm{G}$ mobile systems. Therefore, some of these transition technologies might become part of future 6G mobile systems offering an ultimate user experience while satisfying not yet predicted future applications, use cases, and services that will become widespread in the coming years.

\section{Methods}

This section addresses the methods and experimental procedure used to carry out the measurements. OFDM signals with quadrature phase-shift keying (QPSK) modulation are generated by the bespoke baseband unit (BBU) and transmitted over the setup link. The OFDM signal has 4096 subcarriers spaced at $240 \mathrm{kHz}$ from each other, which results in a signal aggregate bandwidth up to $800 \mathrm{MHz}$. The OFDM signal is compliant with the 5G New Radio standard. Two network scenarios are considered: optical back-to-back 
and end-to-end. For the optical back-to-back scenario, both I\&Q signals are transmitted over two different cores of a prototype of a $10 \mathrm{~km}$ multicore fiber (MCF); the signals are then fed to a pair of photodetectors (PD) and the output (electrical) signals are loopedback into the BBU. For the end-to-end scenario, a $4 \mathrm{~m} 60 \mathrm{GHz}$ mmWave wireless link is added between the PDs and the BBU. The I\&Q signals at the outputs of the two PDs are fed into a V-band transmitter module, which includes antenna and RF modulator. At the receiver side, the received wireless signal is down-converted (from V-band to I\&Q baseband) by the V-band receiver module. Finally, the I\&Q signals are sent back into the BBU for analysis. The signal analysis is shown in the form of constellation diagrams, error vector magnitude, and signal-to-noise ratio.

\subsection{Constellation diagram}

The modulation format (QPSK) and the aggregate signal bandwidth for the analysis of the constellation diagram of the received signal are chosen via the software of the bespoke baseband unit (BBU) before the signal generation and transmission. The BBU transmits up to $1.5 \mathrm{~Gb} / \mathrm{s} 5 \mathrm{G}$ NR OFDM signals with a total of 4096 subcarriers, spaced at $240 \mathrm{kHz}$ from each other, which results in a signal aggregate bandwidth up to $800 \mathrm{MHz}$. For the sake of visualization and simplicity, the subcarriers of the OFDM signal are split into 8 different subcarrier groups, each covering ca. $100 \mathrm{MHz}$. The constellation diagram data is collected for both network scenarios, i.e., back-to-back and end-to-end optical and wireless links.

\subsection{Error vector magnitude (EVM)}

With the same setup, the OFDM signal is modulated with QPSK as well as generated and transmitted by the BBU. The EVM values are obtained for different OFDM subcarrier groups by the BBU software. The EVM data is collected for both network scenarios, i.e., back-to-back and end-to-end optical and wireless links.

\subsection{Signal-to-noise ratio (SNR)}

With the same setup, the OFDM signal the SNR data is collected by the BBU software for the OFDM subcarrier groups and for both network scenarios, i.e., back-to-back and end-to-end optical and wireless links.

\subsection{Radio frequency spectrum}

With the same setup, the signal generated by the BBU is analyzed by the RF analyzer software of the BBU. This signal is captured at the digital-to-analog converter (DAC) output of the transmitter of the BBU.

\footnotetext{
Abbreviations

Al: Artificial intelligence; A-RoF: Analog radio-over-fiber; ADC: Analog to digital converter; BBUs: Baseband units; BS: Base station; C-RAN: Cloud radio access network; eMBB: Enhanced mobile broadband; EVM: Error vector magnitude; FSO: Free-space optics; IBN: Intent-based networking; IQoF: Intensity and quadrature over fiber; KPIs: Key-performance indicators; LED: Light emitting diode; LNA: Low noise amplifier; OFDM: Orthogonal frequency division multiplexing; RU: Remote unit; SDM: Space-division multiplexing; SDN: Software-defined networking; MZM: Mach-Zender modulator; mMTC: Massive machine type communications; MEC: Mobile edge computing; MIMO: Multiple input multiple output;
} 
mmWave: Millimeter wave; MCFs: Multi-core fibers; NFV: Network function virtualization; NOS: Network operating system; NBIs: Northbound interfaces; O/E: Optical to electrical conversion; PD: Photodiode; PA: Power amplifier; ROADM: Reconfigurable optical add-drop multiplexer; THz: Terahertz; URLLC: Ultra-reliable low latency communications; UDWDM: Ultra dense wavelength division multiplexing; VRAN: Virtualized radio access network; QPSK: Quadrature phase-shift keying.

\section{Acknowledgements}

The authors would like to thank all the partners of both the blueSPACE and 5G-PHOS consortia with especial thanks to Siklu, Eulambia, ICOM, Optoscribe and UPV.

\section{Authors' contributions}

The views and ideas discussed in the paper are the result of joint work by all the authors. T.R.R. led the writing of the manuscript and launched the initial idea. S.R. designed the experiment to which all authors contributed. B.C. and S.R. performed analysis of the experimental data. All authors read and approved the final manuscript.

\section{Funding}

This work was partially funded by the blueSPACE and 5G-PHOS 5G-PPP phase 2 projects, which have received funding from the European Union's Horizon 2020 programme under Grant Agreements Number 762055 and 761989. D. PerezGalacho acknowledges the funding of the Spanish Science Ministry through the Juan de la Cierva programme.

\section{Availability of data and materials}

Data sharing is not applicable to this article as only initial datasets were analyzed during the current study.

\section{Declarations}

\section{Competing interests}

The authors declare that they have no competing interests.

\section{Author details}

${ }^{1}$ Institute for Photonic Integration, Eindhoven University of Technology, 5600 MB Eindhoven, The Netherlands. ${ }^{2}$ Present Address: Engineering, Modeling, and Applied Social Sciences Center, Federal University of ABC, Santo André, Brazil. ${ }^{3}$ Department of Informatics, Aristotle University of Thessaloniki, 54124 Thessaloníki, Greece. ${ }^{4}$ Photonics Research Labs, iTEAM Research Institute, Universitat Politecnica de Valencia, Camino de Vera, 46022 Valencia, Spain. ${ }^{5}$ Eulambia Advanced Technologies, Agia Paraskevi, 15342 Athens, Greece. ${ }^{6}$ Intracom Telecom, Markopoulou Avenue, 19002 Peania, Athens, Greece. ${ }^{7}$ Optoscribe Ltd, Rosebank Technology Park, Livingston EH54 7EJ, United Kingdom. ${ }^{8}$ Athens Information Technology, Kifissias Avenue 44, 15125 Marousi, Athens, Greece.

Received: 23 December 2019 Accepted: 30 March 2021

Published online: 21 April 2021

\section{References}

1. L. Zhang, Y. Liang, D. Niyato, 6 G visions: mobile ultra-broadband, super internet-of-things, and artificial intelligence. China Commun. 16(8), 1-14 (2019)

2. W. Saad, M. Bennis, M. Chen, A vision of $6 \mathrm{G}$ wireless systems: applications, trends, technologies, and open research problems. IEEE Netw. 34(3), 134-142 (2020). https://doi.org/10.1109/MNET.001.1900287

3. K.B. Letaief, W. Chen, Y. Shi, J. Zhang, Y.A. Zhang, The roadmap to 6G: Al empowered wireless networks. IEEE Commun. Mag. 57(8), 84-90 (2019)

4. S.J. Nawaz, S.K. Sharma, S. Wyne, M.N. Patwary, M. Asaduzzaman, Quantum machine learning for 6G communication networks: state-of-the-art and vision for the future. IEEE Access 7, 46317-46350 (2019)

5. S. Rommel, T.R. Raddo, U. Johannsen, C. Okonkwoa, I. Tafur Monroy, in Proceedings of SPIE Photonics West. Beyond 5G-Wireless Data Center Connectivity (San Francisco, 2019)

6. P.T. Dat, A. Kanno, N. Yamamoto, T. Kawanishi, Seamless convergence of fiber and wireless systems for $5 \mathrm{G}$ and beyond networks. IEEE/OSA J. Lightwave Technol. 37(2), 592-605 (2019)

7. Z. Zhang et al., $6 \mathrm{G}$ wireless networks: vision, requirements, architecture, and key technologies. IEEE Veh. Technol. Mag. 14(3), 28-41 (2019)

8. P. Yang, Y. Xiao, M. Xiao, S. Li, 6G wireless communications: vision and potential techniques. IEEE Netw. 33(4), 70-75 (2019)

9. T.S. Rappaport et al., Wireless communications and applications above $100 \mathrm{GHz}$ : opportunities and challenges for 6G and beyond. IEEE Access 7, 78729-78757 (2019)

10. A.D. Giglio, A. Pagano, Scenarios and economic analysis of fronthaul in $5 \mathrm{G}$ optical networks. OSA/IEEE J. Lightwave Technol. 37(2), 585-591 (2019)

11. B. Madani, M. Ndiaye, in Proceedings of the 8th International Conference on Modeling Simulation and Applied Optimization (ICMSAO). Autonomous vehicles delivery systems classification: introducing a TSP with a moving depot (Manama 2019)

12. $5 \mathrm{G}$ Infrastructure Association (5G-IA), 5G pan-European trials roadmap v4.0, Press Release (2018)

13. P. Chanclou, L. A. Neto, K. Grzybowski, Z. Tayq, F. Saliou, N. Genay, Mobile fronthaul architecture and technologies: A RAN equipment assessment [invited]. IEEE/OSA J. Opt. Commun. Netw. 10(1), A1-A7 (2018). https://doi.org/10. 1364/JOCN.10.0000A1 
14 I. A. Alimi, A. L. Teixeira and P. P. Monteiro, Toward an efficient C-RAN optical fronthaul for the future networks: a tutorial on technologies, requirements, challenges, and solutions. IEEE Commun. Surv. Tutor. 20(1), 708-769. https://doi. org/10.1109/COMST.2017.2773462

15. M. Xiao, S. Mumtaz, Y. Huang, L. Dai, Y. Li, M. Matthaiou, G.K. Karagiannidis, E. Bjornson, K. Yang, I. Chih-Lin, A. Ghosh, Millimeter wave communications for future mobile networks. IEEE J. Sel. Areas Commun. 35(9), 1909-1935 (2017)

16 S. Qiu, K. Luo, T. Jiang, Beam selection for mmWave massive MIMO systems under hybrid transceiver architecture. IEEE Commun. Lett. 22, 1498-1501 (2018). https://doi.org/10.1109/LCOMM.2018.2829482

17 S. Wu, L. Chiu, J. Wang, Reconfigurable hybrid beamforming for dual-polarized mmWave MIMO channels: stochastic channel modeling and architectural adaptation methods. IEEE Trans. Commun. 66(2), 741-755 (2018). https://doi. org/10.1109/TCOMM.2017.2762689

18. I. Tafur Monroy, T.R. Raddo, S. Rommel, C. Okonkwo, N. Calabretta, U. Johannsen, G. Dubbelman, J. Scholtes, B. Rutten, in Proceedings of the IEEE ICTON. Testing facilities for end-to-end test of vertical applications enabled by $5 \mathrm{G}$ networks: Eindhoven $5 \mathrm{G}$ brainport testbed (Bucharest, 2018)

19. A. El Mahjoubi, T. Mazri, N. Hmina, in Proceedings of the ICWNMC. M2M and eMTC communications via NB-IoT, Morocco first testbed experimental results and RF deployment scenario: new approach to improve main $5 \mathrm{G} \mathrm{KPIs}$ and performances (Rabat 2017)

20. S. Sun, T.S. Rappaport, M. Shafi, in Proceedings of the IEEE Conference on Computer Communications Workshop. Hybrid beamforming for $5 \mathrm{G}$ millimeter-wave multi-cell networks (Honolulu, 2018)

21. L. A. Neto, J. Maes, P. Larsson-Edefors, J. Nakagawa, K. Onohara and S. J. Trowbridge, Considerations on the use of digital signal processing in future optical access networks. J. Lightwave Technol. 38(3), 598-607. https://doi.org/10. 1109/JLT.2019.2946687

22. T.R. Raddo, S. Rommel, C. Vagionas, G. Kalfas, N. Pleros, I. Tafur Monroy, in Proceedings of the European Conference on Networks and Communications. Analog radio-over-fiber 5G fronthaul systems: blueSPACE and 5G-PHOS projects convergence (Valencia, 2019)

23. C. Mitsolidou, C. Vagionas, A. Mesodiakaki, P. Maniotis, G. Kalfas, A. Miliou, N. Pleros, C.G.H. Roeloffzen, P.W.L. van Dijk, R.M. Oldenbeuving, in Proceedings of the EuCNC. A 5G C-RAN architecture for Hot-Spots: OFDM based Analog IFoF PHY and MAC layer design (Valencia, 2019)

24. Y. Amma. Y. Sasaki, K. Takenaga, S. Matsuo, J. Tu, K. Saitoh, M. Koshiba, T. Morioka, Y. Miyamoto, in Proceedings of the Optical Fiber Communication Conference. High-density multicore fiber with heterogeneous core arrangement (Los Angeles, 2015)

25 N. Gkatzios, M. Anastasopoulos, A. Tzanakaki et al., Efficiency gains in $5 \mathrm{G}$ softwarised radio access networks. J. Wirel. Commun. Netw. 2019, 183 (2019). https://doi.org/10.1186/s13638-019-1488-z

26 S. Imtiaz, G. Koudouridis, H. Ghauch et al., Random forests for resource allocation in $5 \mathrm{G}$ cloud radio access networks based on position information. J. Wirel. Commun. Netw. 2018, 142 (2018). https://doi.org/10.1186/ s13638-018-1149-7

27 M.D. Renzo, M. Debbah, D. Phan-Huy et al., Smart radio environments empowered by reconfigurable Al metasurfaces: an idea whose time has come. J. Wirel. Commun. Netw. 2019, 129 (2019). https://doi.org/10.1186/ s13638-019-1438-9

28. R. Gerzaguet, N. Bartzoudis, L.G. Baltar et al., The $5 \mathrm{G}$ candidate waveform race: a comparison of complexity and performance. J. Wirel. Commun. Netw. 2017, 13 (2017)

29 R. Ferrus, O. Sallent, J. Perez-Romero et al. On the automation of RAN slicing provisioning: solution framework and applicability examples. J. Wirel. Commun. Netw. 2019, 167 (2019). https://doi.org/10.1186/s13638-019-1486-1

30 G. Interdonato, E. Bjornson, H. Quoc Ngo et al. Ubiquitous cell-free Massive MIMO communications. J. Wirel. Commun. Netw. 2019, 197 (2019). https://doi.org/10.1186/s13638-019-1507-0

31 B. Wang, L. Peng, P. Ho. Energy-efficient radio-over-fiber system for next-generation cloud radio access networks. J. Wirel. Commun. Netw. 2019, 118 (2019). https://doi.org/10.1186/s13638-019-1457-6

32 V.C.M. Borges, K.V. Cardoso, E. Cerqueira et al. Aspirations, challenges, and open issues for software-based $5 \mathrm{G}$ networks in extremely dense and heterogeneous scenarios. J. Wirel. Commun. Netw. 2015, 164 (2015). https://doi.org/ 10.1186/s13638-015-0380-8

33. X. Liu et al., Efficient mobile fronthaul via DSP-based channel aggregation. OSA/IEEE J. Lightwave Technol. 32(6), 1550-1556 (2016)

34 S. Khatibi, L. Caeiro, L.S. Ferreira et al. Modelling and implementation of virtual radio resources management for $5 \mathrm{G}$ Cloud RAN. J. Wirel. Commun. Netw. 2017, 128 (2017). https://doi.org/10.1186/s13638-017-0908-1

35 M. Peuster, M. Marchetti, G. García de Blas et al. Automated testing of NFV orchestrators against carrier-grade multiPoP scenarios using emulation-based smoke testing. J. Wirel. Commun. Netw. 2019, 172 (2019). https://doi.org/10. 1186/s13638-019-1493-2

36 D. Sabella, P. Serrano, G. Stea et al. Designing the 5 G network infrastructure: a flexible and reconfigurable architecture based on context and content information. J. Wirel. Commun. Netw. 2018, 199 (2018). https://doi.org/10.1186/ s13638-018-1215-1

37 A. Abdelkader, E. Jorswieck. Robust adaptive distributed beamforming for energy-efficient network flooding. J. Wirel. Commun. Netw. 2019, 154 (2019). https://doi.org/10.1186/s13638-019-1434-0

38. S. Maimaiti, G. Chuai, W. Gao et al. A low-complexity algorithm for the joint antenna selection and user scheduling in multi-cell multi-user downlink massive MIMO systems. J. Wirel. Commun. Netw. 2019, 208 (2019). https://doi.org/ 10.1186/s13638-019-1529-7

39. J. Capmany, J. Mora, I. Gasulla, J. Sancho, J. Lloret, S. Sales, Microwave photonic signal processing. OSA/IEEE J. Lightwave Technol. 31, 571-586 (2013)

40. I. Gasulla, D. Barrera, J. Hervas et al., Spatial division multiplexed microwave signal processing by selective grating inscription in homogeneous multicore fibers. Sci. Rep. 7, 41727 (2017)

41. L.R. Chen, Silicon photonics for microwave photonics applications. OSA/IEEE J. Lightwave Technol. 35, 824-835 (2017) 
42. C. Zhang, K. Ota, J. Jia, M. Dong, Breaking the blockage for big data transmission: gigabit road communication in autonomous vehicles. IEEE Commun. Mag. 56(6), 152-157 (2018)

43. A.S. Cacciapuoti, K. Sankhe, M. Caleffi, K.R. Chowdhury, Beyond 5G: THz-based medium access protocol for mobile heterogeneous networks. IEEE Commun. Mag. 56(6), 110-115 (2018)

44. K.M.S. Huq, J.M. Jornet, W.H. Gerstacker, A. Al-Dulaimi, Z. Zhou, J. Aulin, THz communications for mobile heterogeneous networks. IEEE Commun. Mag. 56(6), 94-95 (2018)

45. H. Elayan, O. Amin, R.M. Shubair, M. Alouini, in Proceedings of the International Conference on Advanced Communication Technologies and Networking. Terahertz communication: the opportunities of wireless technology beyond $5 \mathrm{G}$ (Marrakech, 2018)

46. A. Jurado-Navas, J.M. Garrido-Balsells, M. Castillo-Vázquez, A. García-Zambrana, A. Puerta-Notario, in Proceedings of the OFC. Converging underwater and FSO ground communication links (San Diego, 2019)

47. A. Jurado-Navas, T.R. Raddo, J.M. Garrido-Balsells, B.-H.V. Borges, J.J. Vegas Olmos, I. Tafur Monroy, Hybrid optical CDMA/FSO communications network under spatially correlated gamma-gamma scintillation. OSA Opt. Express 24(15), 16799-16814 (2016)

48. A. Jurado-Navas, A. Tatarczak, X. Lu, J.J. Vegas Olmos, J.M. Garrido-Balsells, I. Tafur Monroy, 850-nm hybrid fiber/freespace optical communications using orbital angular momentum modes. Opt. Express 23, 33721-33732 (2015)

49. A. Jurado-Navas, J.M. Garrido-Balsells, J.F. Paris, M. Castillo-Vazquez, A. Puerta-Notario, General analytical expressions for the bit error rate of atmospheric optical communication systems. Opt. Lett. 36, 4095-4097 (2011)

50. A. Jurado-Navas, J.M. Garrido-Balsells, M. Castillo-Vázquez, A. Puerta-Notario, Closed-form expressions for the lower-bound performance of variable weight multiple pulse-position modulation optical links through turbulent atmospheric channels. IET Commun. 6, 390-397 (2011)

51. V. Dhasarathan, M. Singh, J. Malhotra, Development of high-speed FSO transmission link for the implementation of 5G and Internet of Things. Wirel. Netw 26, 2403-2412 (2020)

52. G. Pan, E. Ekici, Q. Feng, Capacity analysis of log-normal channels under various adaptive transmission schemes. Electron. Lett. 6(3), 346-348 (2012)

53. X. Feng, H. Jiang, Z. Wu, T. Wang, H. Jiang, S. Gao, 60 Gbit/s coherent wavelength-division multiplexing free-space optical modulating retro-reflector in a turbulence-tunable atmospheric cell. Opt. Commun. 448, 111-115 (2019)

54. E. Illi, F.E. Bouanani, F. Ayoub, in Proceedings of the International Conference on Wireless Networks and Mobile Communications (WINCOM). A performance study of a hybrid 5G RF/FSO transmission system (Rabat, 2017)

55. H. Huang, G. Xie, Y. Yan, N. Ahmed, Y. Ren, Y. Yue, D. Rogawski, M.J. Willner, B.I. Erkmen, K.M. Birnbaum, 100 Tbit/s free-space data link enabled by three-dimensional multiplexing of orbital angular momentum, polarization, and wavelength. Opt. Lett. 39(2), 197-200 (2014)

56. E. Ciaramella, Y. Arimoto, G. Contestabile, M. Presi, A. D'Errico, V. Guarino, M. Matsumoto, 1.28 Terabit/s (32×40 Gb/s) WDM transmission ver a double-pass free space optical link. IEEE J. Sel. Areas Commun. 27, 1639-1645 (2009)

57. G. Parca, A. Shahpari, V. Carrozzo, G. TosiBeleffi, A.J. Teixeira, Optical wireless transmission at 1.6-tbit/s (16×100 Gbit/s) for next-generation convergent urban infrastructures. Opt. Eng. 52, 116102 (2013)

58. A.T. Pham, P.V. Trinh, V.V. Mai, N.T. Dang, C.-T. Truong, in Proceedings of the Opto-Electronics and Communications Conference (OECC). Hybrid free-space optics/millimeter-wave architecture for $5 \mathrm{G}$ cellular backhaul networks (Shanghai, 2015)

59. P.T. Dat, A. Kanno, K. Inagaki, T. Umezawa, N. Yamamoto, T. Kawanishi, Hybrid optical wireless-mmWave: ultra highspeed indoor communications for beyond 5G, in IEEE INFOCOM 2019-IEEE Conference on Computer Communications Workshops (INFOCOM WKSHPS), Paris, France (2019), pp. 1003-1004. https://doi.org/10.1109/INFCOMW.2019. 8845283

60. D. Nguyen, J. Bohata, M. Komanec, S. Zvanovec, B. Ortega, Z. Ghassemlooy, Seamless 25 GHz transmission of LTE 4/16/64-QAM signals over hybrid SMF/FSO and wireless link. J. Lightwave Technol. 37(24), 6040-6047 (2019). https://doi.org/10.1109/JLT.2019.2945588

61. D. Nguyen, J. Bohata, J. Spacil, D. Dousek, M. Komanec, S. Zvanovec, Z. Ghassemlooy, B. Ortega, M-QAM transmission over hybrid microwave photonic links at the K-band. Opt. Express 27, 33745-33756 (2019)

62. J. Liu, P. Wang, X. Zhang, Y. He, X. Zhou, H. Ye, Y. Li, Xu. Shixiang, S. Chen, D. Fan, Deep learning based atmospheric turbulence compensation for orbital angular momentum beam distortion and communication. Opt. Express 27, 16671-16688 (2019)

63. S. Lohani, R.T. Glasser, Generative machine learning for robust free-space communication. arXiv:1909.02249 (2019)

64. E.J. Shin, V.W.S. Chan, in Proceedings of the Global Communications Conference. Optical communication over the turbulent atmospheric channel using spatial diversity (Taipei, 2002)

65. R. Munoz et al., in Proceedings of the European Conference on Networks and Communications (EuCNC). SDN/ NFV 5 G fronthaul networks integrating analog/digital RoF, optical beamforming, power over fiber and optical SDM technologies (Valencia, 2019)

66. G. Kalfas et al., Next generation fiber-wireless fronthaul for $5 \mathrm{G}$ mmWave networks. IEEE Commun. Mag. 57(3), 138-144 (2019)

\section{Publisher's Note}

Springer Nature remains neutral with regard to jurisdictional claims in published maps and institutional affiliations. 